\title{
DIVERGENCE AND QUASIMORPHISMS OF RIGHT-ANGLED ARTIN GROUPS
}

\author{
JASON BEHRSTOCK AND RUTH CHARNEY
}

\begin{abstract}
We give a group theoretic characterization of geodesics with superlinear divergence in the Cayley graph of a right-angled Artin group $A_{\Gamma}$ with connected defining graph. We use this to determine when two points in an asymptotic cone of $A_{\Gamma}$ are separated by a cut-point. As an application, we show that if $\Gamma$ does not decompose as the join of two subgraphs, then $A_{\Gamma}$ has an infinite-dimensional space of non-trivial quasimorphisms. By the work of Burger and Monod, this leads to a superrigidity theorem for homomorphisms from lattices into right-angled Artin groups.
\end{abstract}

\section{INTRODUCTION}

The divergence of a geodesic, $\gamma:[-\infty, \infty] \rightarrow X$, in a metric space, can roughly be thought of as the growth rate of a function from $\mathbb{N}$ to $\mathbb{R}$, which for each $N \in \mathbb{N}$ gives the length of the shortest path in $X \backslash B_{N}(\gamma(0))$ from $\gamma(-N)$ to $\gamma(N)$, where $B_{N}(\gamma(0))$ is the open ball of radius $N$ about $\gamma(0)$. We refer to the divergence of a finitely generated group to mean the largest divergence over all geodesics in a Cayley graph of $G$.

The divergence function has proven to be a useful tool in studying the large scale geometry of groups. Gersten classified geometric 3-manifolds by their divergence Ger which allows one to distinguish quasi-isometry classes of 3-manifold groups containing hyperbolic pieces from graph manifold groups $\mathrm{KL}$. In addition, divergence functions are closely related to cut-points in the asymptotic cones of a group. Interest in the existence of such cut-points arose from Druţu-Osin-Sapir's result that a group is relatively hyperbolic with respect to a collection of subgroups $\mathcal{H}$ if and only if every asymptotic cone has a collection of cut-points with the property that the maximal subsets of the asymptotic cone not separated by any one of these cut-points arise from asymptotic cones of the subgroups $\mathcal{H}$ [DS.

On the other hand, cut-points in asymptotic cones also arise in groups which are not relatively hyperbolic. To prove that any point in an asymptotic cone of a mapping class group is a cut-point, the first author showed that axes of pseudoAnosov elements in a mapping class group have super-linear divergence. This also implies that these directions are quasi-geodesically stable, or equivalently, Morse geodesics. Beh]. (Alternate proofs have since been obtained by [DMS and DR]). More recently, Druţu-Mozes-Sapir showed in DMS that a group has superlinear divergence if and only if its asymptotic cones contain cut-points, and that this occurs if and only if the group contains Morse geodesics.

Date: January 2010.

R. Charney was partially supported by NSF grant DMS 0705396.

J. Behrstock was partially supported by NSF grant DMS 0812513 . 
In this paper we discuss divergence in right-angled Artin groups. Given a finite, simplicial graph $\Gamma$, the right-angled Artin group $A_{\Gamma}$ is the finitely presented group with generators corresponding to vertices of $\Gamma$ and relators of the form $x^{-1} y^{-1} x y$ whenever the vertices $x$ and $y$ of $\Gamma$ are connected by an edge. Right-angled Artin groups form a rich family of groups interpolating between $\mathbb{Z}^{n}$, the group corresponding to the complete graph on $n$ vertices, and the free group $F_{n}$, corresponding to the graph with $n$ vertices and no edges.

If $\Gamma_{1}$ and $\Gamma_{2}$ are two graphs, their join is the graph obtained by connecting every vertex of $\Gamma_{1}$ to every vertex of $\Gamma_{2}$ by an edge. Subgraphs of $\Gamma$ that decompose as joins are central to understanding divergence of geodesics. We define a notion of join length of a geodesic, which measures the number of cosets of join subgroups the geodesic passes through (see Section 3 for the precise definition) and we prove,

Theorem 4.4 (Divergence and join length). Let $\Gamma$ be a connected graph and let $\alpha$ be a bi-infinite geodesic in $A_{\Gamma}$. Then $\alpha$ has linear divergence if and only if the join length of $\alpha$ is finite.

The proof uses the action of $A_{\Gamma}$ on a $\mathrm{CAT}(0)$ cube complex, $X_{\Gamma}$, the universal cover of the Salvetti complex of $A_{\Gamma}$. We show that the join length of a geodesic $\alpha$ determines the behavior of the walls in $X_{\Gamma}$ crossed by $\alpha$.

From the divergence theorem, we obtain the following complete characterization of when two points in an asymptotic cone of a right-angled Artin group can be separated by a cut-point.

Theorem 4.6 (Classification of pieces). Let $\Gamma$ be a connected graph. Fix a pair of points $\boldsymbol{a}, \boldsymbol{b} \in A_{\Gamma}^{\omega}$. The following are equivalent.

(1) No point of $A_{\Gamma}^{\omega}$ separates $\boldsymbol{a}$ from $\boldsymbol{b}$.

(2) There exist points $\boldsymbol{a}^{\prime}, \boldsymbol{b}^{\prime}$ arbitrarily close to $\boldsymbol{a}, \boldsymbol{b}$, respectively for which the join length between $\boldsymbol{a}^{\prime}, \boldsymbol{b}^{\prime}$ is finite.

In the terminology of DS, cut-points in an asymptotic cone give rise to a treegrading whose pieces are the maximal subsets that cannot be separated by a point. The above result gives a complete description of the pieces in $A_{\Gamma}^{\omega}$. Since rightangled Artin groups are not relatively hyperbolic [BDM], these pieces do not arise by taking asymptotic cones of subgroups of $A_{\Gamma}$ DS.

In BDM, Behrstock-Drutu-Mosher introduce a notion of algebraic thickness of a group. Theorem 4.6 shows that for a connected graph $\Gamma, A_{\Gamma}$ is algebraically thick of order zero if $\Gamma$ is a join, and otherwise it is algebraically thick of order at least one with respect to the set of maximal join subgroups. It was established in BDM, Corollary 10.8], that, except for $\mathbb{Z}$, right-angled Artin groups with connected presentation graph are thick of order at most one. Together, these two results show that if $A_{\Gamma}$ is a join, then it is algebraically thick of order exactly zero, and otherwise it is algebraically thick of order exactly one.

Our main application of divergence is to show that subgroups of right-angled Artin groups have many non-trivial quasi-morphisms. A function $\phi: G \rightarrow \mathbb{R}$ is a homogeneous quasimorphism if $\phi\left(g^{n}\right)=n \phi(g)$ for all $n>0$, and there exists a constant $D \geq 0$ such that

$$
|\phi(g h)-\phi(g)-\phi(h)| \leq D
$$

for every $g, h \in G$. The vector space of homogeneous quasimorphisms, modulo the subspace of true homomorphisms, is denoted $\widetilde{Q H}(G)$ and is related to the bounded 
cohomology of $G$. Bestvina and Fujiwara BF2] have shown that for group actions on a $\mathrm{CAT}(0)$ space, satisfying a weak discontinuity property, the existence of rankone isometries (i.e., hyperbolic isometries with an axis not bounding a half-plane) gives rise to non-trivial quasimorphisms. Using their results we prove,

Theorem 5.2 (Rank-one geodesics and Quasimorphisms). If $G \subseteq A_{\Gamma}$ is any non-cyclic, finitely generated subgroup which is not contained in a conjugate of a join subgroup, then $G$ contains an element which acts as a rank-one isometry of $X_{\Gamma}$. In this case, $\widetilde{Q H}(G)$ is infinite dimensional.

Right-angled Artin groups have been shown to have an extremely rich family of subgroups, cf. $\mathrm{BB}, \mathrm{HW}, \mathrm{CW}$. In contrast, using Theorem 5.2 and BurgerMonod's result on nonexistence of quasimorphisms on higher rank lattices BM1, BM2, Mon1, we deduce:

Corollary 5.3 (Superrigidity with RAAG image). Let $\Lambda$ be an irreducible lattice in a connected semisimple Lie group with finite center, no compact factors, and rank at least 2. Then for any right-angled Artin group $A_{\Gamma}$, every homomorphism $\rho: \Lambda \rightarrow A_{\Gamma}$ is trivial.

To the best of our knowledge this is the most general statement of superrigidity for right-angled Artin groups, although many special cases follow from other methods. For example, for lattices satisfying Kazhdan's Property (T), NibloReeves [NR] showed that every action on a finite dimensional CAT $(0)$ cube complex has a global fixed point. Since $A_{\Gamma}$ acts freely on the cube complex $X_{\Gamma}$, any homomorphism of such a lattice into $A_{\Gamma}$ must be trivial. For non-uniform lattices, superrigidity follows from the Margulis Normal Subgroup Theorem Mar, Zim, since every solvable subgroup of $A_{\Gamma}$ is virtually abelian. Other special cases follow from the work of Shalom [Sha], Monod [Mon2], and Gelander-Karlsson-Margulis GKM.

From Theorem 5.2 we also deduce the following, which P. Dani informed us she has independently established in joint work with A. Abrams, N. Brady, M. Duchin, A. Thomas and R. Young.

Corollary 5.4 (Quadratic divergence). Let $\Gamma$ be a connected graph. $A_{\Gamma}$ has linear divergence if and only if $\Gamma$ is a join; otherwise its divergence is quadratic.

The authors would like to thank Koji Fujiwara and Yehuda Shalom for helpful conversations. Also, Behrstock would like to thank Brandeis University and Charney would like to thank the Forschungsinstitut für Mathematik at ETH, Zurich for their hospitality during the development of this paper.

\section{WALLS}

Let $\Gamma$ be a finite, simplicial graph with vertex set $V$. The right-angled Artin group associated to $\Gamma$ is the group $A_{\Gamma}$ with presentation

$$
\left.A_{\Gamma}=\langle V| v w=w v \text { if } v \text { and } w \text { are connected by an edge in } \Gamma\right\rangle .
$$

Associated to any right-angled Artin group $A_{\Gamma}$ is a $\mathrm{CAT}(0)$ cubical complex $X_{\Gamma}$ with a free action of $A_{\Gamma}$. In this section we describe $X_{\Gamma}$ and investigate the structure of walls in this complex.

Let $T$ be a torus of dimension $|V|$ with edges labelled by the elements of $V$. Let $\bar{X}_{\Gamma}$ denote the subcomplex of $T$ consisting of all faces whose edge labels span a 
complete subgraph in $\Gamma$ (or equivalently, mutually commute in $A_{\Gamma}$ ). $\bar{X}_{\Gamma}$ is called the Salvetti complex for $A_{\Gamma}$. It is easy to verify that the Salvetti complex has fundamental group $A_{\Gamma}$ and that the link of the unique vertex is a flag complex. It follows that its universal cover, $X_{\Gamma}$, is a $\operatorname{CAT}(0)$ cube complex with a free, cocompact action of $A_{\Gamma}$.

If $\Gamma^{\prime}$ is a full subgraph of $\Gamma$, then the inclusion $\Gamma^{\prime} \rightarrow \Gamma$ induces an injective homomorphism $\mathcal{A}_{\Gamma^{\prime}} \rightarrow A_{\Gamma}$ and an embedding $X_{\Gamma^{\prime}} \rightarrow X_{\Gamma}$. This embedding is locally geodesic, and hence (since $X_{\Gamma} \mathrm{CAT}(0)$ ) it is globally geodesic. We may thus view $X_{\Gamma^{\prime}}$ as a convex subspace of $X_{\Gamma}$.

We now recall some basic facts about walls in a CAT(0) cube complex. A wall (or hyperplane) in a $\operatorname{CAT}(0)$ cube complex, $X$, is an equivalence class of midplanes of cubes where the equivalence relation is generated by the rule that two midplanes are related if they share a face. Each wall is a geodesic subspace of $X$ and separates $X$ into two components. Moreover, if a wall contains a (positive length) segment of a geodesic $\gamma$, then it contains the entire geodesic $\gamma$.

In the discussion that follows, we are interested in the relation between nonintersecting pairs of walls. The following terminology will be convenient.

Definition 2.1. Two walls $H_{1}, H_{2}$ in a CAT(0) cube complex are strongly separated if $H_{1} \cap H_{2}=\emptyset$ and no wall intersects both $H_{1}$ and $H_{2}$.

Consider this definition in the context of a right-angled Artin group $A_{\Gamma}$ and the cube complex $X_{\Gamma}$. For example, suppose $\Gamma$ consists of two disjoint edges, so $A_{\Gamma}$ is the free product $\mathbb{Z}^{2} * \mathbb{Z}^{2}$. In this case, the Salvetti complex is the wedge of two tori, and its universal cover $X_{\Gamma}$ consists of flats which pairwise intersect in at most one vertex. Since walls never contain vertices of $X_{\Gamma}$, they remain entirely in one flat. It follows that two walls are strongly separated if and only if they lie in different flats.

At the other extreme, suppose $\Gamma$ is a square, in which case $A_{\Gamma}=F_{2} \times F_{2}$, the product of two free groups of rank 2 , and $X_{\Gamma}$ is the product of two trees $T_{1} \times T_{2}$. The walls consist of trees of the form $T_{1} \times\{y\}$ and $\{x\} \times T_{2}$ where $x$ and $y$ are midpoints of edges in $T_{1}$ and $T_{2}$ respectively. It is now easy to see that no two walls are strongly separated.

Now let $A_{\Gamma}$ be an arbitrary right-angled Artin group and let $H_{1}$ and $H_{2}$ be two walls in $X_{\Gamma}$. Consider the set of all minimal length geodesics from $H_{1}$ to $H_{2}$. It follows from $\mathrm{BH}$, Proposition II.2.2] that the union of all such paths forms a convex subspace of $X_{\Gamma}$ which we call the bridge between $H_{1}$ and $H_{2}$.

Lemma 2.2. If $H_{1}$ and $H_{2}$ are strongly separated, then the bridge $B$ between them consists of a single geodesic from $\mathrm{H}_{1}$ to $\mathrm{H}_{2}$.

Proof. It suffices to show that $B \cap H_{1}$ (and by symmetry $B \cap H_{2}$ ) is a single point. We first show that $B \cap H_{1}$ does not intersect any other wall $H$. For suppose $x \in B \cap H_{1} \cap H$. Since $x \in B$, it is the initial point of some minimal length geodesic $\gamma$ from $H_{1}$ to $H_{2}$. The initial segment of $\gamma$ lies in some cube $\sigma$ of $X_{\Gamma}$ which contains midplanes in both $H_{1}$ and $H$. These midplanes span $\sigma$, hence the initial segment of $\gamma$, which is orthogonal to $H_{1}$, must lie in $H$. It follows that all of $\gamma$ lies in $H$ and hence $H \cap H_{2} \neq \emptyset$. This contradicts the assumption that $H_{1}$ and $H_{2}$ are strongly separated. 
Now every wall $H$ has an open neighborhood $N(H)$ isometric to $H \times(0,1)$, namely the neighborhood consisting of the interiors of all cubes containing a midplane in $H$. Then the same argument as above (using parallel copies of $H$ in $N(H)$ ) shows that $B \cap H_{1} \cap N(H)$ must also be empty for all $H \neq H_{1}$. The only convex subsets of $H_{1}$ disjoint from every $N(H)$ are single vertices, so it follows that $B \cap H_{1}$ is a single point.

Lemma 2.3. There are universal constants $C, D>1$ (depending only on the dimension of $X_{\Gamma}$ ) such that if $H_{1}$ and $H_{2}$ are strongly separated and $B$ is the bridge between them, then

(1) for any $x \in H_{1}$ and $y \in H_{2}$,

$$
d(x, y) \geq C^{-1}(d(x, B)+d(y, B))-d\left(H_{1}, H_{2}\right)-4
$$

(2) for any geodesic $\alpha$ in $X_{\Gamma}$, and any point $c$ on $\alpha$, if $H_{1}$ and $H_{2}$ intersect $\alpha$ inside the ball of radius $r$ about $c$, then the bridge $B$ is contained in the ball of radius Dr about $c$.

Proof. (1) For any two points $x, y$ in $X_{\Gamma}$, define $d_{H}(x, y)$ to be the number of walls separating $x$ and $y$, or equivalently, the number of walls crossed by a geodesic from $x$ to $y$. This distance function is quasi-isometric to the geodesic metric in $X_{\Gamma}$. More precisely,

$$
d(x, y)-C \leq d_{H}(x, y) \leq C d(x, y)+C
$$

where $C$ is the diameter of a maximal cube.

By Lemma 2.2. $B$ consists of a single geodesic $\gamma$ from $H_{1}$ to $H_{2}$. Let $h_{1}, h_{2}$ be the endpoints of $\gamma$. Let $\alpha$ be the geodesic from $h_{1}$ to $x$, and $\beta$ the geodesic from $y$ to $h_{2}$. Note that $\alpha$ lies in $H_{1}$ and $\beta$ lies in $H_{2}$. Since the strongly separated hypothesis guarantees that no wall crosses both $\alpha$ and $\beta$, the path $\alpha \gamma \beta$ crosses any given wall at most twice and $d_{H}(x, y)$ is the number of walls it crosses exactly once. It follows that

$$
d_{H}(x, y) \geq d_{H}\left(x, h_{1}\right)+d_{H}\left(y, h_{2}\right)-d_{H}\left(h_{1}, h_{2}\right) .
$$

Applying the inequalities above, we obtain

$$
\begin{aligned}
d(x, y) & \geq C^{-1} d_{H}(x, y)-1 \\
& \geq C^{-1}\left(d_{H}\left(x, h_{1}\right)+d_{H}\left(y, h_{2}\right)-d_{H}\left(h_{1}, h_{2}\right)\right)-1 \\
& \geq C^{-1}\left(d\left(x, h_{1}\right)+d\left(y, h_{2}\right)-2 C\right)-d\left(h_{1}, h_{2}\right)-2 \\
& \geq C^{-1}(d(x, B)+d(y, B))-d\left(H_{1}, H_{2}\right)-4 .
\end{aligned}
$$

(2) Suppose $x=H_{1} \cap \alpha$ and $y=H_{2} \cap \alpha$ are in the ball of radius $r$ about $c$. Then every point in $B$ is within $k=\frac{1}{2}(d(x, B)+d(y, B))+d\left(H_{1}, H_{2}\right)$ of either $x$ or $y$ and hence within $k+r$ of $c$. By part $(1), d(x, B)+d(y, B)$ is bounded by a linear function of $d(x, y)$, and by hypothesis, $d\left(H_{1}, H_{2}\right) \leq d(x, y) \leq 2 r$ so $k$ is bounded by a linear function of $r$.

We now introduce the notion of divergence for bi-infinite geodesics and discuss how the existence of strongly separated walls affects the divergence.

Definition 2.4. Let $X$ be a geodesic metric space. Let $\alpha: \mathbb{R} \rightarrow X$ be a bi-infinite geodesic in $X$ and let $\rho$ be a linear function $\rho(r)=\delta r-\lambda$ with $0<\delta<1$ and $\lambda \geq 0$. Define $\operatorname{div}(\alpha, \rho)(r)=$ length of the shortest path from $\alpha(-r)$ to $\alpha(r)$ which stays outside the ball of radius $\rho(r)$ about $\alpha(0)$ (or $\operatorname{div}(\alpha, \rho)(r)=\infty$ if no such 
path exists). We say $\alpha$ has linear divergence if for some choice of $\rho, \operatorname{div}(\alpha, \rho)(r)$ is bounded by a linear function of $r$, and that $\alpha$ has super-linear divergence otherwise.

It is not difficult to verify that the definition of linear divergence is independent of the choice of basepoint $\alpha(0)$. We leave this as an exercise for the reader.

Theorem 2.5. Let $\alpha$ be a bi-infinite geodesic in $X_{\Gamma}$ and let $\mathcal{H}$ be the sequence of walls crossed by $\alpha$. Suppose $\mathcal{H}$ contains an infinite subsequence $\left\{H_{0}, H_{1}, \ldots\right\}$ such that for all $i, H_{i}$ is strongly separated from $H_{i+1}$. Then $H_{i}$ is strongly separated from $H_{j}$ for all $i \neq j$ and $\alpha$ has superlinear divergence.

Proof. Let $\alpha^{+}$and $\alpha^{-}$denote the limit points of $\alpha$ in $\partial X_{\Gamma}$. Since $H_{i}$ is disjoint from $H_{i+1}$, the half spaces $H_{i}^{+}$containing $\alpha^{+}$form a directed set $H_{0}^{+} \subset H_{1}^{+} \subset \ldots$. Hence no two of these walls intersect and if some wall $H$ intersects both $H_{i}$ and $H_{j}, i<j$, then it must cross $H_{i+1}$, contradicting the strong separation of $H_{i}$ from $H_{i+1}$. It follows that $H_{i}$ and $H_{j}$ are strongly separated for any $i<j$.

Let $r^{\prime}=\rho(r)$ and consider the ball $B_{r^{\prime}}$ of radius $r^{\prime}$ about $\alpha(0)$. Let $x_{i}=H_{i} \cap \alpha$. By Lemma 2.3 for any $n$, we can choose $r$ large enough so that $B_{r^{\prime} / 2}$ contains $x_{i}$ for all $i \leq n$, as well as the bridge between $H_{i-1}$ and $H_{i}$. Let $\beta$ be any path from $\alpha(-r)$ to $\alpha(r)$ which stays outside $B_{r^{\prime}}$. Then $\beta$ must cross $H_{0}, H_{1}, \ldots, H_{n}$ in a sequence of points $y_{0}, y_{1}, \ldots y_{n}$. Note that each $y_{i}$ is distance at least $r^{\prime} / 2$ from the bridges to the adjacent walls, hence by Lemma 2.3 , there is a universal constant $C$ such that

$$
\begin{aligned}
d\left(y_{i-1}, y_{i}\right) & \geq \frac{r^{\prime}}{C}-\left(d\left(H_{i-1}, H_{i}\right)-4\right. \\
& \geq \frac{r^{\prime}}{C}-d\left(x_{i-1}, x_{i}\right)-4
\end{aligned}
$$

It follows that the length of $\beta$ satisfies

$$
\begin{aligned}
|\beta| & \geq \sum_{n r^{\prime}} d\left(y_{i-1}, y_{i}\right) \\
& \geq \frac{n r^{\prime}}{C}-4 n-d\left(x_{0}, x_{n}\right) \\
& \geq \frac{n r^{\prime}}{C}-4 n-r^{\prime}
\end{aligned}
$$

Since $n \rightarrow \infty$ as $r \rightarrow \infty$, this proves the superlinear divergence of $\alpha$.

The following example shows that the converse of the above theorem does not hold in complete generality. However, when $\Gamma$ is a connected graph, we will give a complete characterization of geodesics with superlinear divergence in Theorem 4.4 below.

Example 2.6. Suppose $\Gamma$ is disconnected, then $A_{\Gamma}$ splits as a free product and $X_{\Gamma}$ splits as a wedge of spaces. Take any point $p \in X_{\Gamma}$ whose removal disconnects $X_{\Gamma}$, and any pair of geodesic rays $\gamma_{1}$ and $\gamma_{2}$ emanating from $p$ for which $\gamma_{1} \backslash\{p\}$ and $\gamma_{2} \backslash\{p\}$ are in distinct components of $X_{\Gamma} \backslash\{p\}$. Then the union of $\gamma_{1}$ and $\gamma_{2}$ is a bi-infinite geodesic with super-linear divergence (indeed infinite divergence, since $\gamma_{1}$ and $\gamma_{2}$ can not be connected in the complement of any ball around $p$ ). If we choose each of the $\gamma_{i}$ to be contained in (the cube neighborhood of) a wall, then $\alpha=\gamma_{1} \cup \gamma_{2}$ is a geodesic with superlinear divergence such that no three walls crossed by $\alpha$ are pairwise strongly separated.

\section{JOINS}

In this section we give a group-theoretic interpretation of Theorem 2.5 Choosing a vertex $x_{0}$ in $X_{\Gamma}$ as a basepoint, we may identify the 1-skeleton of $X_{\Gamma}$ with the Cayley graph of $A_{\Gamma}$ so that vertices are labeled by elements of $A_{\Gamma}$ and edges by 
elements of the standard generating set (namely the vertex set of $\Gamma$ ). For a generator $v$, let $e_{v}$ denote the edge from the basepoint 1 to the vertex $v$. Any edge in $X_{\Gamma}$ determines a unique wall, namely the wall containing the midpoint of that edge. Denote by $H_{v}$ the wall containing the midpoint of $e_{v}$.

For a cube in $X_{\Gamma}$, all of the parallel edges are labelled by the same generator $v$. It follows that all of the edges crossing a wall $H$ have the same label $v$, and we call this a wall of type $v$. Since $A_{\Gamma}$ acts transitively on edges labeled $v$, a wall is of type $v$ if and only if it is a translate of the standard wall $H_{v}$.

Let $l k(v)$ denote the subgraph of $\Gamma$ spanned by the vertices adjacent to $v$ and $s t(v)$ the subgraph spanned by $v$ and $l k(v)$. The stabilizer of the wall $H_{v}$ is the group generated by $l k(v)$, which we denote by $L_{v}$. To see this, note that in any cube containing the edge $e_{v}$, all other edges labeled $v$ are of the form $g e_{v}$ for some $g \in L_{v}$. An induction on the number of cubes between $e_{v}$ and $e$ now shows that the same holds for any edge $e$ which crosses $H_{v}$.

In what follows, for two subgroups $K$ and $L$ of $A_{\Gamma}$, we will use the notation $K L$ to mean the set of elements of $A_{\Gamma}$ which can be written as a product $k l$ for some $k \in K, l \in L$. In general, $K L$ is not a subgroup.

Lemma 3.1. Let $H_{1}=g_{1} H_{v}$ and $H_{2}=g_{2} H_{w}$. Then

(1) $H_{1}$ intersects $H_{2} \Longleftrightarrow v, w$ commute and $g_{1}^{-1} g_{2} \in L_{v} L_{w}$.

(2) $\exists H_{3}$ intersecting both $H_{1}$ and $H_{2} \Longleftrightarrow \exists u \in \operatorname{st}(v) \cap \operatorname{st}(w)$ such that $g_{1}^{-1} g_{2} \in L_{v} L_{u} L_{w}$.

Here (2) includes the case in which $H_{3}$ is equal to $H_{1}$ or $H_{2}$, hence $H_{1}$ and $H_{2}$ are strongly separated if and only if the conditions in (2) are not satisfied.

Proof. Without loss of generality, we may assume that $H_{1}=H_{v}$ and $H_{2}=g H_{w}$.

(1) If $v, w$ commute, they span a cube in $X_{\Gamma}$, hence $H_{v}$ and $H_{w}$ intersect. Suppose $g=a b$, with $a \in L_{v}, b \in L_{w}$. Then $H_{v}=a^{-1} H_{v}$ and $H_{w}=b H_{w}$, so translating by $a$, we see that $H_{v}$ intersects $g H_{w}$.

Conversely, suppose $H_{v}$ intersects $g H_{w}$ in a cube $C$. Then $C$ contains edges of type $v$ and of type $w$ hence $v$ and $w$ must commute. Moreover, $C$ is a translate $C=h C^{\prime}$ of a cube $C^{\prime}$ at the basepoint containing the edges $e_{v}$ and $e_{w}$. Since $e_{v}$ and $h e_{v}$ both intersect $H_{v}, h$ lies in $L_{v}$. Since $g e_{w}$ and $h e_{w}$ both intersect $g H_{w}$, $h^{-1} g$ lies in $L_{w}$. Thus, $g \in L_{v} L_{w}$.

(2) If $u \in \operatorname{st}(v) \cap s t(w)$ and $g=a b c \in L_{v} L_{u} L_{w}$, then $H_{v}$ and $b H_{w}=b c H_{w}$ both intersect $H_{u}=b H_{u}$. Translating by $a$, we see that $H_{v}$ and $g H_{w}$ both intersect $a H_{u}$.

Conversely, suppose that $H_{3}=h H_{u}$ intersects both $H_{1}$ and $H_{2}$. By part (1), u must commute with both $v$ and $w$, so $u \in s t(v) \cap s t(w)$. Also by part (1), $h \in L_{v} L_{u}$ and $h^{-1} g \in L_{u} L_{w}$, so $g \in L_{v} L_{u} L_{w}$.

For two walls $H_{v}$ and $g H_{w}$ to satisfy the conditions of (2), both $w$ and the letters in $g$ must lie in a 2 -neighborhood of $v$. The converse is not true. Consider for example the case of the Artin group associated to a pentagon $\Gamma$ with vertices labeled (in cyclic order) $a, b, c, d, e$. Every vertex lies in a 2-neighborhood of $a$, but we claim that $H_{a}$ and $d a H_{c}$ are strongly separated. This follow from the lemma since $s t(a) \cap s t(c)=\{b\}$ and $d a$ does not lie in $L_{a} L_{b} L_{c}=\langle e, b\rangle\langle a, c\rangle\langle b, d\rangle$.

To guarantee that no two walls in $X_{\Gamma}$ are strongly separated, we need a stronger condition. Let $\Gamma_{1}$ and $\Gamma_{2}$ be (non-empty) graphs. The join of $\Gamma_{1}$ and $\Gamma_{2}$ is the graph formed by joining every vertex of $\Gamma_{1}$ to every vertex of $\Gamma_{2}$ by an edge. The Artin group associated to such a graph splits as a direct product, $A_{\Gamma}=A_{\Gamma_{1}} \times A_{\Gamma_{2}}$ 
and $X_{\Gamma}$ splits as a metric product $X_{\Gamma}=X_{\Gamma_{1}} \times X_{\Gamma_{2}}$. The walls in $X_{\Gamma}$ are thus of the form $H_{1} \times X_{\Gamma_{2}}$ or $X_{\Gamma_{1}} \times H_{2}$ for some wall $H_{i}$ in $X_{\Gamma_{i}}$. Clearly, every wall of the first type intersects every wall of the second type, and it follows that no two walls are strongly separated.

Let $g \in A_{\Gamma}$ and let $v_{1} v_{2} \ldots v_{k}$ be a minimal length word representing $g$. For $i<k$, set $g_{i}=v_{1} v_{2} \ldots v_{i}$. Then the set of walls crossed by the edge path in $X_{\Gamma}$ from $x_{0}$ to $g x_{0}$ labelled $v_{1} v_{2} \ldots v_{k}$ is given by

$$
\mathcal{H}=\left\{H_{v_{1}}, g_{1} H_{v_{2}}, g_{2} H_{v_{3}} \ldots g_{k-1} H_{v_{k}}\right\} \text {. }
$$

A different choice of minimal word gives the same set of walls, changing only the order in which they are crossed. Define the separation length of $g$ to be

$\ell_{S}(g)=\max \{k \mid \mathcal{H}$ contains $k$ walls which are pairwise strongly separated $\}$.

If $J$ is a complete subgraph of $\Gamma$ which decomposes as a non-trivial join, then we call $A_{J}$ a join subgroup of $A_{\Gamma}$. Define the join length of $g$ to be

$$
\ell_{J}(g)=\min \left\{k \mid g=a_{1} \ldots a_{k} \text { where } a_{i} \text { lies in a join subgroup of } A_{\Gamma}\right\} .
$$

If $\alpha$ is a (finite) geodesic in $A_{\Gamma}$, we can approximate $\alpha$ by an edge path which crosses the same set of walls as $\alpha$. The word labeling this edge path determines an element $g_{\alpha} \in A_{\Gamma}$. We define $\ell_{S}(\alpha)=\ell_{S}\left(g_{\alpha}\right)$ and $\ell_{J}(\alpha)=\ell_{J}\left(g_{\alpha}\right)$. If $\alpha$ is a bi-infinite geodesic, and $\alpha_{n}$ denotes the restriction of $\alpha$ to the interval $[-n, n]$, we define the separation and join lengths of $\alpha$ to be

$$
\ell_{S}(\alpha)=\lim _{n \rightarrow \infty} \ell_{S}\left(\alpha_{n}\right) \quad \ell_{J}(\alpha)=\lim _{n \rightarrow \infty} \ell_{J}\left(\alpha_{n}\right) .
$$

Lemma 3.2. A bi-infinite geodesic $\alpha$ in $A_{\Gamma}$ has finite join length if and only if both the positive and negative rays of $\alpha$ eventually stay in a single join. If every bi-infinite periodic geodesic in $A_{\Gamma}$ has finite join length, then $\Gamma$ is itself a join.

Proof. For any join $J$ in $\Gamma, X_{J}$ is a convex subspace of $X_{\Gamma}$ so once $\alpha$ leaves $X_{J}$, it will never return, and similarly for translates of $X_{J}$. If $\alpha$ has finite join length it lies entirely in some finite set of these join subspaces and hence each ray must eventually remain in a single join. The reverse implication is obvious.

For the second statement, suppose $\Gamma$ is not a join. Let $J$ be a maximal join in $\Gamma$ and let $v$ be a vertex not in $J$. Let $g \in A_{J}$ be the product of all the vertices in $J$ and consider the bi-infinite geodesic $\alpha=\ldots$ gvgvgvgv ... Note that no vertex $w \in J \cup v$ commutes with both $J$ and $v$ since otherwise, we would have $J \cup v$ contained in the join $s t(w)$, contradicting the maximality of $J$. It follows that the tails of $\alpha$ must involve every vertex of $J \cup v$, hence by the first statement of the lemma, $\alpha$ has infinite join length.

In the proof of the previous lemma, we used the fact that for any vertex $v$ of $\Gamma, \operatorname{st}(v)$ is always a join, namely it is the join of $\{v\}$ and $l k(v)$. This fact plays a crucial role in the next lemma as well as in the proof of Theorem 4.4 below.

Lemma 3.3. For any $g \in A_{\Gamma}$,

$$
\ell_{S}(g) \leq \ell_{J}(g) \leq 2 \ell_{S}(g)+1
$$

Thus a bi-infinite geodesic has infinite join length if and only if it has infinite separation length. 
Proof. The first inequality follows from the observation above that no two walls in a join are strongly separated. For the second inequality, fix a minimal word for $g$ and let $\mathcal{H}$ be the sequence of walls crossed by the corresponding edge path as listed above. Set $H=H_{v_{1}}$ and let $H^{\prime}=g_{i} H_{v_{i+1}}$ be the first wall in the sequence strongly separated from $H$. Then by Lemma 3.1 $g_{i}$ lies in the product of three link subgroups, $L_{v_{1}} L_{u_{1}} L_{v_{i+1}}$, for some $u_{1}$, hence $g_{i+1}=g_{i} v_{i+1}$ lies in a product of the three join groups generated by $s t\left(v_{1}\right), s t\left(u_{1}\right)$, and $s t\left(v_{i+1}\right)$. Now repeat this argument starting with $H=g_{i} H_{v_{i+1}}$ and taking $H^{\prime}=g_{j} H_{v_{j+1}}$ to be the next strongly separated wall (or $H^{\prime}=$ the last wall in $\mathcal{H}$ if no more strongly separated walls exist), to conclude that $g_{j+1}$ lies in the product of join subgroups

$$
\left\langle s t\left(v_{1}\right)\right\rangle\left\langle s t\left(u_{1}\right)\right\rangle\left\langle s t\left(v_{i+1}\right)\right\rangle\left\langle s t\left(u_{2}\right)\right\rangle\left\langle s t\left(v_{j+1}\right)\right\rangle .
$$

Continuing this process, each new strongly separated wall adds two star subgroups. Since we encounter at most $\ell_{S}(g)$ strongly separated walls, the inequality follows.

\section{The ASYmptotic CONE}

The goal of this section is to understand the structure of the asymptotic cones of $A_{\Gamma}$. We begin by recalling some preliminaries on asymptotic cones, tree graded spaces, and divergence; we refer the reader to [DW], DS, and [DMS] for more details.

Let $(X, d)$ be a geodesic metric space. Let $\omega$ be a non-principal ultrafilter, $\left(o_{n}\right)$ a sequence of observation points in $X$, and $\left(d_{n}\right)$ a sequence of scaling constants such that $\lim _{\omega} d_{n}=\infty$. Then the asymptotic cone, Cone $\omega\left(X,\left(o_{n}\right),\left(d_{n}\right)\right)$, is the metric space consisting of equivalence classes of sequences $\left(a_{n}\right)$ satisfying $\lim _{\omega} d\left(o_{n}, a_{n}\right) / d_{n}<\infty$, where two such sequences $\left(a_{n}\right),\left(a_{n}^{\prime}\right)$ represent the same point $\boldsymbol{a}$ if and only if $\lim _{\omega} d\left(a_{n}, a_{n}^{\prime}\right) / d_{n}=0$, and the metric is given by $d_{\omega}(\boldsymbol{a}, \boldsymbol{b})=\lim _{\omega} d\left(a_{n}, b_{n}\right) / d_{n}$.

We will assume the observation points and scaling constants are fixed and write $X^{\omega}$ for Cone $_{\omega}\left(X,\left(o_{n}\right),\left(d_{n}\right)\right)$. In general, $X^{\omega}$ is a complete geodesic metric space. In the case where $X$ has a cocompact group action, $X^{\omega}$ is independent of choice of observation points (but not, in general, of scaling constants) and is homogeneous.

A complete geodesic metric space is tree graded if it contains a collection of closed subsets, $\mathcal{P}$, called pieces such that the following three properties are satisfied: in each $P \in \mathcal{P}$, every pair of points is connected by a geodesic in $P$; any simple geodesic triangle is contained in some $P \in \mathcal{P}$; and each pair $P, P^{\prime} \in \mathcal{P}$ is either disjoint or intersects in a single point. Druţu-Osin-Sapir proved that a group is relatively hyperbolic if and only if all of its asymptotic cones are tree-graded with respect to pieces obtained by taking asymptotic cones of the peripheral subgroups. On the other hand, Behrstock-Drutu-Mosher proved that right-angled Artin groups are relatively hyperbolic if and only if their defining graph is disconnected [BDM]. In this section, we show that for connected defining graphs, although the Artin group $A_{\Gamma}$ is not relatively hyperbolic, the asymptotic cones of $A_{\Gamma}$ still have a non-trivial tree grading providing $\Gamma$ is not a join. Moreover, although the pieces do not come from asymptotic cones of subgroups, they can be characterized group-theoretically (see Theorem 4.6).

We begin by recalling the work of Drutu-Mozes-Sapir [DMS] on divergence and cut-points. 
Definition 4.1. Let $\rho(k)=\delta k-\lambda$ with $0<\delta<1$ and $\lambda \geq 0$. For points $a, b, c \in X$, set $k=d(c,\{a, b\}))$ and define $\operatorname{div}(a, b, c ; \rho)$ to be the length of the shortest path in $X$ from $a$ to $b$ which lies outside the ball of radius $\rho(k)$ about $c$. The divergence of $X$ with respect to $\rho$ is the function

$$
\operatorname{Div}(X, \rho)(r)=\sup \{\operatorname{div}(a, b, c ; \rho) \mid a, b \in X, d(a, b) \leq r\} .
$$

For a biinfinite geodesic $\alpha$, the divergence function introduced in Section 2 can be written as,

$$
\operatorname{div}(\alpha, \rho)(r)=\operatorname{div}(\alpha(-r), \alpha(r), \alpha(0) ; \rho) .
$$

In particular, if $X$ has linear divergence, then every bi-infinite geodesic in $X$ has linear divergence.

Drutu-Mozes-Sapir establish the following correspondence between cut-points and divergence functions [DMS, Lemma 3.14].

Proposition 4.2 (DMS). Let $\boldsymbol{a}=\left(a_{n}\right), \boldsymbol{b}=\left(b_{n}\right), \boldsymbol{c}=\left(c_{n}\right)$ be three points in $X^{\omega}$, and let $k=d_{\omega}(\boldsymbol{c},\{\boldsymbol{a}, \boldsymbol{b}\})$. Then $\boldsymbol{c}$ is a cut-point separating $\boldsymbol{a}$ from $\boldsymbol{b}$ if and only if for some $\rho$,

$$
\frac{\lim _{\omega} \operatorname{div}\left(a_{n}, b_{n}, c_{n} ; \frac{\rho}{k}\right)}{d_{n}}=\infty .
$$

In particular, for a bi-infinite geodesic $\alpha$ in $X$, taking $a_{n}=\alpha\left(-d_{n}\right), b_{n}=\alpha\left(d_{n}\right)$, and $c_{n}=\alpha(0)$, the proposition implies that $\boldsymbol{c}$ is a cut-point separating $\boldsymbol{a}$ from $\boldsymbol{b}$ if and only if $\alpha$ has super-linear divergence.

We say that $X$ is wide if no asymptotic cone of $X$ has cut-points. In the case that $X$ is the Cayley graph of a group $G$, the proposition above leads to the following criterion for $G$ to be wide (see [DMS, Proposition 1.1]).

Proposition 4.3 (DMS $).$ A group $G$ is wide if and only if Div $(G, \rho)(r)$ is linear for $\rho(r)=\frac{1}{2} r-2$.

In the case of $A_{\Gamma}$, the divergence of a bi-infinite geodesic is determined by its join length.

Theorem 4.4 (Divergence and join length). Let $\Gamma$ be a connected graph and let $\alpha$ be a bi-infinite geodesic in $X_{\Gamma}$. Then $\alpha$ has linear divergence if and only if the join length of $\alpha$ is finite.

Proof. If the join length of $\alpha$ is infinite, then by Lemma 3.3. so is the separation length. By Theorem 2.5, it follows that $\alpha$ has super-linear divergence.

Now suppose that the join length of $\alpha$ is finite. We will show that $\alpha$ lies in a subspace of $X_{\Gamma}$ whose image in any asymptotic cone $X_{\Gamma}^{\omega}$ has no cut-points. It then follows from the remarks following Proposition 4.2 that $\alpha$ has linear divergence.

By Lemma 3.2, $\alpha$ lives entirely in a finite union of join subspaces, that is, subspaces which are translates of $X_{J}$ for some join $J$. Since $A_{J}$ decomposes as a direct product of infinite groups, $X_{J}$ is wide. Hence in any asymptotic cone $X_{\Gamma}^{\omega}$, the cone on $g X_{J}$ gives rise to a subspace with no cut-points. If $g^{\prime} X_{J^{\prime}}$ is another join subspace which shares a geodesic line with $g X_{J}$, then the union of their asymptotic cones contains a line in $X_{\Gamma}^{\omega}$ hence also has no cut-points.

Thus, it suffices to show that any two join subspaces $g X_{J}$ and $g^{\prime} X_{J^{\prime}}$ are connected by a sequence of join subspaces such that consecutive subspaces share a line. We will call this a connecting sequence. By hypothesis, the graph $\Gamma$ is connected, 
so we can find a sequence of joins beginning at $J$ and ending at $J^{\prime}$ such that consecutive joins in the sequence share at least one vertex in $\Gamma$. (For example, take a path from $J$ to $J^{\prime}$ and take the sequence of stars of the vertices along this path.) For $g=g^{\prime}$, it follows that there is a connecting sequence from $g X_{J}$ to $g X_{J}^{\prime}$.

For the general case, we may assume without loss of generality that $g=1$. Say $g^{\prime}=a_{1} \ldots a_{k}$ where each $a_{i}$ lies in some join $J_{i}$. Then the observation above shows that there are connecting sequences from $X_{J}$ to $X_{J_{1}}\left(=a_{1} X_{J_{1}}\right)$, from $a_{1} X_{J_{1}}$ to $a_{1} X_{J_{2}}\left(=a_{1} a_{2} X_{J_{2}}\right)$, and so on to $g^{\prime} X_{J_{k}}$, and finally, from $g^{\prime} X_{J_{k}}$, to $g^{\prime} X_{J^{\prime}}$.

We now generalize the notion of join length to points in the asymptotic cone $A_{\Gamma}^{\omega}$. For two points $\boldsymbol{a}, \boldsymbol{b} \in A_{\Gamma}^{\omega}$, we say that the pair $\boldsymbol{a}, \boldsymbol{b}$ (or the geodesic between them) has finite join length if there exist representative sequences $\left(a_{n}\right),\left(b_{n}\right)$ for which $\lim _{\omega} \ell_{J}\left(a_{n}^{-1} b_{n}\right)<\infty$.

Lemma 4.5. Let $\boldsymbol{a}, \boldsymbol{b}$ be distinct points in $A_{\Gamma}^{\omega}$ and let $\boldsymbol{\alpha}$ be the geodesic between them. If $\boldsymbol{c}$ is a point in the interior of $\boldsymbol{\alpha}$ which does not separate $\boldsymbol{a}$ from $\boldsymbol{b}$, then there exists a neighborhood of $\boldsymbol{c}$ in $\boldsymbol{\alpha}$ such that any two points in this neighborhood have finite join length. Moreover, the union of any two such neighborhoods of $\boldsymbol{c}$ also has this property.

Proof. By Proposition 4.2 the hypotheses imply that for some choice of linear function $\rho, \operatorname{div}\left(a_{n}, b_{n}, c_{n} ; \rho / k\right)$ is bounded by a linear function of $d_{n}$. Let $D>0$ be as in Lemma 2.3 and let $k_{n}=d\left(a_{n}, b_{n}\right)$. Since $\lim _{\omega} \frac{k_{n}}{d_{n}}>0$, we can choose $\epsilon>0$ such that $D \epsilon<\frac{\rho\left(k_{n}\right)}{2 d_{n}}$ for $\omega$-almost every $n$. Consider two sequences $\left(a_{n}^{\prime}\right),\left(b_{n}^{\prime}\right)$ lying within $\epsilon d_{n}$ of $\left(c_{n}\right)$. For any two strongly separated walls between $a_{n}^{\prime}$ and $b_{n}^{\prime}$, Lemma 2.3 (2) implies that the bridge between them lies in the ball of radius $\frac{\rho\left(k_{n}\right)}{2}$ about $c_{n}$. The number of such walls must be bounded (independent of $n$ ), since if not, then arguing as in Theorem 2.5. we would deduce that $\operatorname{div}\left(a_{n}, b_{n}, c_{n} ; \rho / k\right)$ was super-linear. It follows from Lemma 3.3 that the join length is also bounded.

To prove the last statement of the lemma, suppose $\boldsymbol{c}$ lies in two such intervals and let $\boldsymbol{a}^{\prime}, \boldsymbol{b}^{\prime}$ be a point in each. Then there exist two representative sequences $\left(c_{n}\right),\left(c_{n}^{\prime}\right)$ for $\boldsymbol{c}$ such that the sequences $\left(a_{n}^{\prime-1} c_{n}\right)$ and $\left(c_{n}^{\prime-1} b_{n}^{\prime}\right)$ have bounded join length. If $\left(c_{n}^{-1} c_{n}^{\prime}\right)$ has infinite join length, then $\operatorname{div}\left(a_{n}^{\prime}, b_{n}^{\prime}, c_{n} ; \rho / k^{\prime}\right) / d_{n}$ is unbounded for every $\rho$, so by Proposition 4.2, $\boldsymbol{c}$ is a cut-point. This contradicts the hypotheses of the lemma, so we conclude that $\left(c_{n}^{-1} c_{n}^{\prime}\right)$, and hence $\left(a_{n}^{\prime-1} b_{n}\right)$, has bounded join length.

Theorem 4.6 (Classification of pieces). Let $\Gamma$ be a connected graph. Fix a pair of points $\boldsymbol{a}, \boldsymbol{b} \in A_{\Gamma}^{\omega}$. The following are equivalent.

(1) No point of $A_{\Gamma}^{\omega}$ separates $\boldsymbol{a}$ from $\boldsymbol{b}$.

(2) There exist points $\boldsymbol{a}^{\prime}, \boldsymbol{b}^{\prime}$ arbitrarily close to $\boldsymbol{a}, \boldsymbol{b}$, respectively for which the join length between $\boldsymbol{a}^{\prime}, \boldsymbol{b}^{\prime}$ is finite.

Proof. Suppose (1) holds. Let $\boldsymbol{\alpha}$ be the geodesic from $\boldsymbol{a}$ to $\boldsymbol{b}$. Then by Lemma4.5 every point on $\boldsymbol{\alpha}$ is contained in an open interval in which any two points have finite join length. Moreover, if two such intervals intersect, then their union also has this property. It now follows easily that the maximal open interval of $\boldsymbol{\alpha}$ such that any two points have finite join length is the entire interior of $\boldsymbol{\alpha}$.

Now suppose (2) holds. By hypothesis, for every $\epsilon>0$ there exist points $\boldsymbol{a}^{\prime}$ and $\boldsymbol{b}^{\prime}$ with representatives $\left(a_{n}^{\prime}\right)$ and $\left(b_{n}^{\prime}\right)$ whose distances in $A_{\Gamma}^{\omega}$ are less than $\epsilon$ from $\boldsymbol{a}$ 
and $\boldsymbol{b}$, respectively, and the join length between $\boldsymbol{a}^{\prime}$ and $\boldsymbol{b}^{\prime}$ is finite, that is, there exists a constant $M$ such that $\omega$-almost every $a_{n}^{\prime-1} b_{n}^{\prime}$ is a product of at most $M$ subwords, each contained in a join subgroup. Hence the corresponding geodesic is contained in a finite sequence of join cosets. As in the proof of Theorem 4.4 there is a connecting sequence, that is, a finite sequence of additional join cosets which we may add between any two of these to get an ordered sequence of join cosets where each intersects the next in an infinite diameter set. Starting with $M$ join cosets, the construction in Theorem 4.4 yields a connecting sequence whose length is at most $M \cdot \operatorname{diam}(\Gamma)$, where $\operatorname{diam}(\Gamma)$ is the diameter of $\Gamma$. Denote this sequence by $\mathcal{S}_{n}$

We will say that a coset of $A_{J}$ has join type $J$. Since there are only finitely many joins in $\Gamma$, the sequence of join types in $\mathcal{S}_{n}$ is the same for $\omega$-almost every $n$. Any two cosets of the same join type are isometric, so for each $i$, the subspace of points $\left(x_{n}\right) \in A_{\Gamma}^{\omega}$ such that $x_{n}$ lies in the $i^{t h}$ term of $S_{n}$ is isometric to $A_{J_{i}}^{\omega}$ for some join $J_{i}$. In particular, this subspace has no cut-points. Moreover, the intersection of any two consecutive subspaces in this sequence has infinite diameter. It follows that their union, which contains $\boldsymbol{a}^{\prime}$ and $\boldsymbol{b}^{\prime}$, has no cut-points.

Hence, $\boldsymbol{a}$ and $\boldsymbol{b}$ can be approximated arbitrarily closely by points $\boldsymbol{a}^{\prime}$ and $\boldsymbol{b}^{\prime}$ which cannot be separated by a point. Since not being separated by a point is a closed condition, this completes the proof that (2) implies (1).

As an immediate corollary we obtain,

Corollary 4.7. $A_{\Gamma}$ is wide if and only if $\Gamma$ decomposes as a non-trivial join.

The following example shows that one cannot replace the second condition in the theorem by the simpler statement that the geodesic from $\boldsymbol{a}$ to $\boldsymbol{b}$ has finite join length.

Example 4.8. Suppose $x, y$ are two vertices in $\Gamma$ that are not contained in a join. For simplicity, take the scaling constants for $A_{\Gamma}^{\omega}$ to be $d_{n}=n$. Let $a_{n}=1$ for all $n$ and let

$$
b_{n}=x^{\left\lfloor\frac{n}{2}\right\rfloor} y^{\left\lfloor\frac{n}{4}\right\rfloor} x^{\left\lfloor\frac{n}{8}\right\rfloor} y^{\left\lfloor\frac{n}{16}\right\rfloor} \ldots
$$

Then the join length of from $\boldsymbol{a}$ to $\boldsymbol{b}$ is infinite. However, if we truncate each $b_{n}$ after $k$ terms, setting

$$
b_{n}^{(k)}=x^{\left\lfloor\frac{n}{2}\right\rfloor} y^{\left\lfloor\frac{n}{4}\right\rfloor} \ldots z^{\left\lfloor\frac{n}{\left.2^{k}\right\rfloor}\right.}
$$

where $z=x, y$ depending on whether $k$ is odd or even, we obtain a point $\boldsymbol{b}^{(k)}$ in the asymptotic cone whose distance from $\boldsymbol{b}$ is $\frac{1}{2^{k}}$ while the join length from $\boldsymbol{a}$ to $\boldsymbol{b}^{(k)}$ is $k$. It follows from the theorem above that $\boldsymbol{a}$ and $\boldsymbol{b}$ lie in the same piece of the asymptotic cone, despite the fact that the join length between them is infinite.

\section{Divergence, QUASIMORPHISMS, AND SUPERRIGIDITY}

Recall that a hyperbolic isometry of a proper $\mathrm{CAT}(0)$ space is called a rank-one isometry if some axis of that isometry does not bound a half-plane. A quasimorphism on a group $G$ is a function $\phi: G \rightarrow \mathbb{R}$ for which there exists a constant $D(\phi) \geq 0$ such that

$$
|\phi(g h)-\phi(g)-\phi(h)| \leq D(\phi)
$$

for every $g, h \in G$. A quasimorphism is homogeneous if for each $g \in G$ and each $n \in \mathbb{N}$, we have $\phi\left(g^{n}\right)=n \phi(g)$. The set of homogeneous quasimorphisms on a given 
group $G$ form a vector space. The quotient of this vector space by homomorphisms from $G$ to $\mathbb{R}$ is denoted $\widetilde{Q H}(G)$ and is isomorphic to the kernel of the map from the second bounded cohomology of $G$ (with $\mathbb{R}$ coefficients) to the second cohomology of $G$. (For details, see Gro and Cal.)

Burger and Monod proved that there are no non-trivial homogeneous quasimorphisms on any irreducible lattice in a connected semisimple Lie group of rank at least 2 with finite center and no compact factors [BM1, BM2, Mon1. On the other hand, several interesting families of groups, including non-elementary hyperbolic groups $\mathrm{EF}$ and mapping class groups $\mathrm{BF}$, have been shown to have infinite dimensional $\widetilde{Q H}$. We now establish where right-angled Artin groups lay in this framework.

To prove the theorem we will need some basic facts about normal forms in right-angled Artin groups. We refer the reader to Lau for details. Let $V$ be the generating set for $A_{\Gamma}$ and let $g$ be an element $A_{\Gamma}$. A reduced word for $g$ is a minimal length word in the free group $F(V)$ representing $g$. Given an arbitrary word representing $g$, one can obtain a reduced word by a process of "shuffling" (i.e. interchanging commuting elements) and canceling inverse pairs. Any two reduced words for $g$ differ only by shuffling. In particular, the support of $g$, that is the set $\operatorname{Supp}(g) \subseteq V$ of letters appearing in a reduced word for $g$, is independent of choice of reduced word.

An element of $A_{\Gamma}$ is called cyclically reduced if it is of minimal length in its conjugacy class. For any $g \in A_{\Gamma}$, there exists a unique cyclically reduced element conjugate to $g$, which we denote by $\bar{g}$. Given a reduced word $w$ representing $g$, we can find a reduced word $\bar{w}$ for $\bar{g}$ by shuffling $w$ to get a maximal length word $u$ such that $w=u \bar{w} u^{-1}$. In particular, $g=\bar{g}$ if and only if every shuffle of $w$ is cyclically reduced as a word in the free group $F(V)$.

Lemma 5.1. Let $g=\bar{g}$ be a cyclically reduced element of $A_{\Gamma}$. Then the following are equivalent.

(1) $g$ is contained in a join subgroup.

(2) The centralizer of $g$ is non-cyclic.

(3) The centralizer of $g$ is contained in a join subgroup.

Proof. (1) implies (2) since the centralizer of any element $\left(g_{1}, g_{2}\right)$ of a direct product $G_{1} \times G_{2}$ is the product of the centralizers $C_{G_{1}}\left(g_{1}\right) \times C_{G_{2}}\left(g_{2}\right)$. (3) implies (1) is obvious, so it remains only to prove that (2) implies (3).

For any subset $S \subset V$, let $l k(S)$ denote the (possibly empty) set of vertices at distance 1 from every vertex of $S$. It follows from Servatius' Centralizer Theorem $\mathrm{Ser}$ (see also Thm 1.2 of [Lau] that the centralizer of a cyclically reduced element $g$ lies in the subgroup generated by $\operatorname{Supp}(g) \cup l k(\operatorname{Supp}(g))$ and that the centralizer is cyclic unless either $l k(\operatorname{Supp}(g))$ is nonempty or $\operatorname{Supp}(g)$ decomposes as a join. In either case, $\operatorname{Supp}(g) \cup l k(\operatorname{Supp}(g))$ spans a join in $\Gamma$.

Theorem 5.2 (Rank-one geodesics and Quasimorphisms). If $G \subseteq A_{\Gamma}$ is any noncyclic, finitely generated subgroup which is not contained in a conjugate of a join subgroup, then $G$ contains an element which acts as a rank-one isometry of $X_{\Gamma}$. In this case, $\widetilde{Q H}(G)$ is infinite dimensional.

Proof. Let $g$ be an element of $A_{\Gamma}$ and let $\bar{g}$ denote the cyclic reduction of $g$. Then $\bar{g}^{k}$ is geodesic for all $k$. If $\bar{g}$ does not lie in a join subgroup, then the axis for $\bar{g}$ 
has infinite join length, hence by Theorem 4.4, it has super-linear divergence. It follows that the axis for $\bar{g}$ cannot bound a half-flat and the same holds for the axis of $g$ since it is a translate of the axis for $\bar{g}$. Thus to prove the first statement of the theorem, it suffices to show that $G$ contains an element $g$ whose cyclic reduction $\bar{g}$ does not lie in a join subgroup.

Choose an element $c \in G$ such that $\operatorname{Supp}(\bar{c})$ is maximal. That is, if $g \in G$ has $\operatorname{Supp}(\bar{c}) \subseteq \operatorname{Supp}(\bar{g})$, then $\operatorname{Supp}(\bar{c})=\operatorname{Supp}(\bar{g})$. Conjugating $G$ if necessary, we may assume without loss of generality that $c=\bar{c}$. If $c$ is not contained in a join, we are done. So suppose $c$, and hence by Lemma 5.1 the centralizer of $c$, is contained in a join subgroup $A_{J}$.

By hypothesis, $G$ does not lie in a join, so there is some element $h \in G$ whose support is not contained in $J$. Consider an element of the form $x=c^{k} h c^{k} \in$ $G$. We claim that for sufficiently large $k, \operatorname{Supp}(\bar{x}) \supsetneq \operatorname{Supp}(c)$ contradicting the maximality assumption on $\operatorname{Supp}(c)$. To see this, note that since $c$ is cyclically reduced, cancellations can only occur between generators in $c$ and generators in $h$. It follows that repeatedly multiplying $h$ on the left or right by $c$, can result in at most finitely many cancellations and all canceled letters must lie in $\operatorname{Supp}(c) \cap \operatorname{Supp}(h)$. Thus for $k$ sufficiently large, a reduced word for $x$ is of the form $c^{i} u c^{j}$ for some $i, j>0$ and some reduced word $u \in G$, and the support of $x$ satisfies $\operatorname{Supp}(x)=$ $\operatorname{Supp}\left(c^{i} u c^{j}\right)=\operatorname{Supp}(c) \cup \operatorname{Supp}(h)$.

Now consider the cyclic reduction $\bar{x}$ of $x$. Write $u=a \bar{u} a^{-1}$, so $x=c^{i} a \bar{u} a^{-1} c^{j}$. Since $c$ is cyclically reduced, the only way $x$ can fail to be cyclically reduced is if some initial subword $a^{\prime}$ of $a$ commutes with $c$. But in this case, we may conjugate $G$ by $a^{\prime-1}$ and repeat the argument replacing $h$ by $h^{\prime}=a^{\prime-1} h a^{\prime}$ to conclude that $x^{\prime}=c^{i} \bar{u} c^{j}$ is cyclically reduced. Since $a^{\prime}$ lies in the centralizer of $c$, it lies in $A_{J}$, whereas $h \notin A_{J}$. It follows that $h^{\prime} \notin A_{J}$, hence $\operatorname{Supp}\left(\bar{x}^{\prime}\right)=\operatorname{Supp}\left(x^{\prime}\right)=$ $\operatorname{Supp}(c) \cup \operatorname{Supp}\left(h^{\prime}\right) \supsetneq \operatorname{Supp}(c)$, as claimed.

For the second statement of the theorem, note that the action of any non-cyclic subgroup of $A_{\Gamma}$ on $X_{\Gamma}$ is weakly properly discontinuous in the sense of BestvinaFujiwara BF1] since it is a properly discontinuous action of a non-virtually cyclic group on a $\mathrm{CAT}(0)$ space. Since the action of $G$ on $X_{\Gamma}$ contains a rank-one isometry, the Main Theorem of BF2 implies that $\widetilde{Q H}(G)$ is infinite dimensional.

Corollary 5.3 (Superrigidity with RAAG image). Let $\Lambda$ be an irreducible lattice in a connected semisimple Lie group with finite center, no compact factors, and rank at least 2. Then for any right-angled Artin group $A_{\Gamma}$, every homomorphism $\rho: \Lambda \rightarrow A_{\Gamma}$ is trivial.

Proof. We proceed by induction on the number $n$ of vertices in $\Gamma$. If $n=1$, then $A_{\Gamma}$ is infinite cyclic and the Margulis Normal Subgroup Theorem Mar, Zim implies that the image of $\rho$ is trivial.

Suppose $n \geq 2$. If the image $\rho(\Lambda)$ is not contained in the conjugate of a join subgroup, then Theorem 5.2 yields a non-trivial quasimorphism on $\rho(\Lambda)$, and hence the composition of this with $\rho$ gives a nontrivial quasimorphism on $\Lambda$. This contradicts Burger-Monod's result that such lattices do not admit any non-trivial quasimorphisms BM1, BM2, Mon1]. 
Thus, up to conjugacy, we may assume that $\rho(\Lambda)$ lies in a join subgroup $A_{J}=$ $A_{\Gamma_{1}} \times A_{\Gamma_{2}}$. Composing $\rho$ with the projections on each factor, gives two homomorphisms from $\Lambda$ to right-angled Artin groups with less than $n$ generators. By induction, both of these homomorphisms are trivial, hence $\rho$ is also trivial.

Corollary 5.4 (Quadratic divergence). Let $\Gamma$ be a connected graph. $A_{\Gamma}$ has linear divergence if and only if $\Gamma$ is a join; otherwise its divergence is quadratic.

Proof. The statement that $A_{\Gamma}$ has linear divergence if and only if $\Gamma$ is a join follows immediately from Proposition 4.3 and Corollary 4.7.

For the second statement, consider the case that $\Gamma$ is not a join. Since $X_{\Gamma}$ is a locally-compact $\mathrm{CAT}(0)$ space, we can apply [KL, Proposition 3.3] which implies that any complete periodic geodesic in $X_{\Gamma}$ has divergence which is either linear or at least quadratic. Theorem 5.2 states that $A_{\Gamma}$ contains a rank-one geodesic, hence the divergence of $A_{\Gamma}$ is at least quadratic.

On the other hand, in [BDM] it is shown that for any connected graph $\Gamma, A_{\Gamma}$ is algebraically thick of order at most 1 . It is not hard to show that any group which is algebraically thick of order 1 has at most quadratic divergence (a generalization of this fact for metric spaces which are thick of arbitrary degree will appear in $\mathrm{BD}$ ). We conclude that the divergence of $A_{\Gamma}$ is exactly quadratic.

\section{REFERENCES}

[Beh] J. Behrstock. Asymptotic geometry of the mapping class group and Teichmüller space. Geometry \&3 Topology 10(2006), 2001-2056.

[BD] J. Behrstock and C. Druţu. Divergence and rigidity of quasi-isometric embeddings. In preparation.

$[\mathrm{BDM}]$ J. Behrstock, C. Druţu, and L. Mosher. Thick metric spaces, relative hyperbolicity, and quasi-isometric rigidity. Math. Ann. 344(2009), 543-595.

[BB] M. Bestvina and N. Brady. Morse theory and finiteness properties of groups. Invent. Math. 129(1997), 445-470.

[BF1] M. Bestvina and K. Fujiwara. Bounded cohomology of subgroups of mapping class groups. Geometry and Topology 6(2002), 69-89 (electronic).

[BF2] M. Bestvina and K. Fujiwara. A characterization of higher rank symmetric spaces via bounded cohomology. Geom. Funct. Anal. 19 (2009), 11-40.

[BH] M. Bridson and A. Haefliger. Metric spaces of non-positive curvature. Springer, 1999.

[BM1] M. Burger and N. Monod. Bounded cohomology of lattices in higher rank Lie groups. $J$. Eur. Math. Soc. (JEMS) 1(1999), 199-235.

[BM2] M. Burger and N. Monod. Continuous bounded cohomology and applications to rigidity theory. Geom. Funct. Anal. 12(2002), 219-280.

[Cal] D. Calegari. scl, volume 20 of MSJ Memoirs. Mathematical Society of Japan, Tokyo, 2009.

[CW] J. Crisp and B. Wiest. Embeddings of graph braid and surface groups in right-angled Artin groups and braid groups. Algebr. Geom. Topol. 4 (2004), 439-472.

[dDW] L. Van den Dries and A. Wilkie. On Gromov's theorem concerning groups of polynomial growth and elementary logic. J. Algebra 89(1984), 349-374.

[DMS] C. Druţu, S. Mozes, and M. Sapir. Divergence in lattices in semisimple Lie groups and graphs of groups. Trans. AMS (2008). To appear. arXiv:0801.4141

[DS] C. Druţu and M. Sapir. Tree-graded spaces and asymptotic cones of groups. Topology 44(2005), 959-1058. With an appendix by Denis Osin and Mark Sapir.

[DR] M. Duchin and K. Rafi. Divergence of geodesics in Teichmuller space and the mapping class group. Geom. Funct. Anal. 19 (2009), 722-742.

[EF] D.B.A. Epstein and K. Fujiwara. The second bounded cohomology of word-hyperbolic groups. Topology 36(1997), 1275-1289.

[GKM] T. Gelander, A. Karlsson, and G.A. Margulis. Superrigidity, generalized harmonic maps and uniformly convex spaces. Geom. Funct. Anal. 17(2008), 1524-1550. 
[Ger] S.M. Gersten. Divergence in 3-manifold groups. Geom. Funct. Anal. 4(1994), 633-647.

[Gro] M. Gromov. Volume and bounded cohomology. IHES Sci. Publ. Math. (1982), 5-99 (1983).

[HW] F. Haglund and D. Wise. Special cube complexes. Geom. Funct. Anal. 17 (2008), no. 5, 1551-1620.

[KL] M. Kapovich and B. Leeb. 3-manifold groups and nonpositive curvature. Geom. Funct. Anal. 8(1998), 841-852.

[Lau] M. Laurence. A generating set for the automorphism group of a graph group. J. London Math. Soc. (2) 52 (1995), no. 2, 318-334.

[Mar] G. A. Margulis. Discrete subgroups of semisimple Lie groups, volume 17 of Ergebnisse der Mathematik und ihrer Grenzgebiete (3) [Results in Mathematics and Related Areas (3)]. Springer-Verlag, Berlin, 1991.

[Mon1] N. Monod. Continuous bounded cohomology of locally compact groups, volume 1758 of Lecture Notes in Mathematics. Springer-Verlag, Berlin, 2001.

[Mon2] N. Monod. Superrigidity for irreducible lattices and geometric splitting. J. Amer. Math. Soc. 19(2006), 781-814 (electronic).

[MSW] L. Mosher, M. Sageev, and K. Whyte. Quasi-actions on trees II: Finite depth Bass-Serre trees. Mem. AMS.

[NR] G. Niblo and L. Reeves. Groups acting on CAT(0) cube complexes. Geom. Topol. 1(1997), $1-7$.

[Ser] H. Servatius. Automorphisms of graph groups. J. Algebra 126 (1989), no. 1, 34-60.

[Sha] Y. Shalom. Rigidity of commensurators and irreducible lattices. Invent. Math. 141(2000), $1-54$.

[Zim] R. Zimmer. Ergodic theory and semisimple groups, volume 81 of Monographs in Math. Birkhauser, 1984. 